\title{
Use of miR-145 and testicular nuclear receptor 4 inhibition to reduce chemoresistance to docetaxel in prostate cancer
}

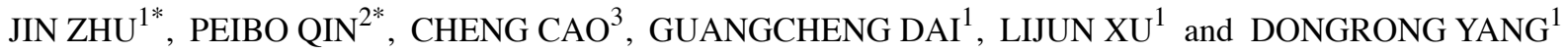 \\ ${ }^{1}$ Department of Urology, The Second Affiliated Hospital of Soochow University, Suzhou, Jiangsu 215004; \\ ${ }^{2}$ Department of Urology, The Affiliated Suzhou Hospital of Nanjing Medical University, Suzhou Municipal Hospital, Suzhou, \\ Jiangsu 215008; ${ }^{3}$ Department of Urology, The First People's Hospital of Changshu, Suzhou, Jiangsu 215500, P.R. China
}

Received July 14, 2020; Accepted November 30, 2020

DOI: $10.3892 /$ or.2021.7925

\begin{abstract}
The human testicular nuclear receptor 4 (TR4) is a critical regulatory gene for the progression of prostate cancer (PCa). Although it has been revealed that TR4 causes chemoresistance in $\mathrm{PCa}$ via the activation of octamer-binding transcription factor 4 (OCT4), the detailed mechanism remains unexplored. In the present study, it was revealed that inhibition of TR4 by shRNA in PCa enhanced the sensitivity to docetaxel in vitro and in vivo. TR4 induced the downregulation of miR-145 by directly binding it to the promoter of miR-145, which was confirmed by chromatin immunoprecipitation analysis and luciferase assay. The overexpression of miR-145 suppressed both the chemoresistance and the expression of OCT4 mRNA and protein. Additionally, the TR4 shRNA mediated re-sensitization to docetaxel, along with the downregulated expression of OCT4, were reversed by the concurrent inhibition of miR-145. The luciferase assay revealed that the activity of the wild-type OCT4 3' untranslated region reporter was suppressed. This suppression diminished when the miR-145 response element mutated. These findings suggest an undescribed regulatory pathway in $\mathrm{PCa}$, by which TR4 directly suppressed the expression of miR-145, thereby inhibiting its direct target OCT4, leading to the promotion of chemoresistance in PCa.
\end{abstract}

\section{Introduction}

Prostate cancer (PCa) is the second leading cause of cancer-associated mortality in men in America and Europe (1).

Correspondence to: Dr Dongrong Yang or Dr Lijun Xu, Department of Urology, The Second Affiliated Hospital of Soochow University, 1055 Sanxiang Road, Suzhou, Jiangsu 215004, P.R. China

E-mail: docydr163@outlook.com

E-mail: xulijun7313079@163.com

${ }^{*}$ Contributed equally

Key words: testicular nuclear receptor 4, docetaxel, prostate cancer, microRNA, chemotherapy
In China, the morbidity and mortality rates of $\mathrm{PCa}$ have been markedly increasing for the last two decades (2). The majority of newly diagnosed PCa patients in China are at an advanced or metastatic stage that can only be treated by androgen deprivation therapy (ADT) instead of radical therapy options such as radical prostatectomy and radical radiotherapy (3). However, the efficacy of ADT eventually decreases and cancer will inevitably develop into castration-resistant prostate cancer (CRPC) (4).

Currently, CRPC can be treated by chemotherapy, immunotherapy, and new androgen receptor blockade agents (5). For example, when CRPC patients were treated with abiraterone and enzalutamide, the overall survival period was extended for 15.8 and 18.4 months, respectively (6). However, the efficacy was primarily dependent on the AR-V7 expression status in the cancer tissue or the circulating cancer cell (7). For instance, several chemotherapeutic agents such as methotrexate, 5-fluorouracil, cyclophosphamide, doxorubicin, and docetaxel, were heavily investigated for the treatment of CPRC before the new androgen receptor blockade agents were used. The results indicated that docetaxel is the most effective agent for CRPC compared with other chemotherapeutic agents (8). Therefore, docetaxel-based chemotherapy is still widely used for the treatment of CRPC (5). The majority of patients exhibit limited disease control and overall survival (9). Most of these patients have become refractory to second-line chemotherapy such as cabazitaxel (10). The overall efficacy of all palliative methods is far from satisfactory.

The mechanism by which CRPC patients display different therapeutic efficacy to docetaxel remains unexplored to this day. It suggested that increased cancer stem cell population (11), dysregulated transcription factors (12), anti-apoptosis factors (13), miRNAs (14), as well as testicular nuclear receptor 4 (TR4) (15) are associated with chemoresistance in CRPC.

MicroRNAs (miRNAs) are small sets of non-coding RNAs that are 21-23 nucleotides in length, which regulate target genes by the degradation of mRNA or by suppressing mRNA translation. miRNAs regulate the initiation, progression, and chemosensitivity of different types of cancers, including prostate cancer. For example, miR-200a has been revealed to modulate chemoresistance by targeting TP53INP1 and YAP1 in breast cancer (16). miR-429 sensitized pancreatic cancer cells to gemcitabine through the negative regulation of 
PDCD4 (17). miRNAs such as miR-200c (18), miR-205 (18), miR-21 (19), and miR-34 (20) have been reported to modulate chemosensitivity in PCa.

Although the transcription of miRNA is non-coding, it is regulated by transcription factors (TFs). It has been revealed that miRNAs and TFs can cooperate to regulate gene expression and biological processes (21). Increasing evidence has indicated that the aberrant regulation of miRNAs by TFs can cause various diseases, including cancer. For example, Li et al reported that HIF1A suppresses the expression of miR-34a in colorectal cancer cells, which in turn promotes the expression of PPP1R11, thereby activating the epithelial-mesenchymal transition (22). Chang et al revealed that miR-137 exerts its pro-metastatic function by targeting TFAP2C in non-small cell lung cancer cells (23). A great amount of recent research has investigated how miRNAs act to regulate their target genes (miRNA-gene regulation) and the role they play in various types of cancer (24). However, studies on how miRNAs are regulated by TFs (TF-miRNA regulation) are quite limited.

TR4 is a transcriptional factor that modulates various molecular signals in multiple malignant tumors including $\mathrm{PCa}(25)$. A previous study has revealed that TR 4 can affect the chemosensitivity of $\mathrm{PCa}$ stem/progenitor cells by regulating octamer-binding transcription factor 4 (OCT4) expression (15). In addition, OCT4 is also regulated by miRNAs to exert its function to affect chemosensitivity (25). The present study focused on whether any 'intermediary miRNA' exists between TR4 and OCT4 to form the TR4/miRNA/OCT4 axis than can regulate chemosensitivity in $\mathrm{PCa}$.

\section{Materials and methods}

Bioinformatics methods. Text mining was used to screen chemoresistance-related miRNAs in PCa. Four online tools including TargetScan (http://targetscan.org), miRDB (http://mirdb.org), miRanda (http://microrna.org), and miRTarBase (http://mirtarbase.mbc.nctu.edu.tw) predicted microRNAs which targeted the human OCT4 gene. Results were merged from each database into one dataset. The JASPAR database (http://jaspar.genereg.net) was utilized to predict the putative TR4 transcription factor binding site of the promoter region of microRNA.

Cell culture. The C4-2 human PCa cell line (from ATCC) was provided by Dr Jer-Tsong Hsieh of the University of Texas Southwestern Medical Center (Dallas, USA). The PC3 and DU-145 human PCa cell lines were purchased from the Cell Bank of Type Culture Collection of the Chinese Academy of Sciences (CBTCCCAS; Shanghai, China). All of the cell lines were verified by STR analysis. PC 3 and $\mathrm{C} 4-2$ cells were maintained in RPMI-1640 medium containing penicillin $(25 \mathrm{U} / \mathrm{ml})$, streptomycin $(25 \mathrm{~g} / \mathrm{ml}), 1 \% \mathrm{~L}$-glutamine and $10 \%$ fetal bovine serum (all from Gibco; Thermo Fisher Scientific, Inc.), at $37^{\circ} \mathrm{C}$ with $5 \% \mathrm{CO}_{2}$ in a humidified atmosphere. The DU-145 cells were maintained in DMEM medium (Gibco; Thermo Fisher Scientific, Inc.) containing penicillin $(25 \mathrm{U} / \mathrm{ml})$, streptomycin $(25 \mathrm{~g} / \mathrm{ml}), 1 \% \mathrm{~L}$-glutamine, and $10 \%$ FBS, at $37^{\circ} \mathrm{C}$ with $5 \% \mathrm{CO}_{2}$ in a humidified atmosphere. After three to four (3-4) passages, the cells grew well and were ready to use in the experiments.
Plasmids and lentivirus. A second-generation lentiviral vector and packing system was used. The TR4 short hairpin (sh) RNA sequence (5'-cgggagaaaccaagcaattg-3') was cloned into pLKO.1 puro plasmid (Addgene, Inc.). In order to overexpress TR4, TR4 cDNA was cloned into the PWPI vector (Addgene, Inc.). Lentivirus packaging and purification were the same as previously described (15). The concentration of the lentiviral vectors was $1 \mu \mathrm{g} / \mu \mathrm{l}$. The $293 \mathrm{~T}$ cell line (ATCC) was used to generate the packaged lentivirus. For transfection, $20 \mu \mathrm{g}$ lentiviral plasmid $(1 \mu \mathrm{g} / \mu \mathrm{l})$ was used, and the ratio of the lentiviral plasmid:packaging vector:envelope was was 2:1:1. The duration of transfection was $48 \mathrm{~h}$. Then conditioned medium containing viral particles was collected, cellular debris was removed, and viral particles were concentrated. Lentivirus expressing or inhibiting hsa-miR-145 or non-targeting negative control was purchased from Shanghai GeneChem Co., Ltd. Primers for cloning were obtained from Sangon Biotech Co., Ltd. Human genomic DNA (Promega Corporation) was used as the template DNA. Lentivirus multiplicity of infection (MOI) for the C-2, PC-3, and DU-145 cell lines was 20 . The duration of transduction into cells of interest was $24 \mathrm{~h}$ and the time interval between transduction and subsequent experimentation was $24 \mathrm{~h}$.

RNA transfection. Chemically synthesized human miR-145 mimic and inhibitor and their corresponding non-targeting negative controls were purchased from Shanghai Genechem Co., Ltd. The sequences were as follows: miR-145-5p mimics sense, 5'-GUCCAGUUUUCCCAGGAAUCCCU-3' and antisense, 5'-GGAUUCCUGGGA AAACUGGACUU-3'; mimics negative control sense, 5'-UUCUCCGAACGUGUC ACGUTT-3' and antisense, 5'-ACGUGACACGUUCGGAGA ATT-3'; miR-145-5p inhibitor, 5'-AGGGAUUCCUGGGAA AACUGGAC-3'; inhibitor negative control, 5'-CAGUAC UUUUGUGUAGUACAA-3'. According to the manufacturer's instructions for transient transfection, cells at $50 \%$ confluence were transfected with miRNA mimics (final concertration $50 \mathrm{nmol} / \mathrm{l}$ ) or inhibitors (final concertration $100 \mathrm{nmol} / \mathrm{l}$ ) using Lipofectamine 3000 (Invitrogen; Thermo Fisher Scientific, Inc.) at room temperature. The duration of transfection was $10 \mathrm{~min}$. The cells were then incubated under $37^{\circ} \mathrm{C}$ for $24 \mathrm{~h}$ before subsequent experimentations.

$R N A$ extraction and reverse transcription-quantitative ( $R T-q)$ $P C R$. For both cells and tissues, total RNAs were isolated using Trizol reagent (Invitrogen; Thermo Fisher Scientific, Inc.). Total RNA ( $2 \mu \mathrm{g})$ was subjected to reverse transcription using Superscript III transcriptase (Invitrogen; Thermo Fisher Scientific, Inc.) according to the manufacturer's instructions. Quantitative real-time PCR (qPCR) was conducted using a Bio-Rad CFX96 system with SYBR-Green (Invitrogen; Thermo Fisher Scientific, Inc.) to determine the mRNA expression level of the target gene. Thermocycling conditions were as follows: Samples were initially held at $95^{\circ} \mathrm{C}$ for $3 \mathrm{~min}$, then processed through 40 cycles at $95^{\circ} \mathrm{C}$ for $15 \mathrm{sec}$ and $60^{\circ} \mathrm{C}$ for $45 \mathrm{sec}$. The $2^{-\Delta \Delta C q}$ method was used for quantification (26). The expression levels of specific genes were normalized by being compared to the expression of GAPDH mRNA. The sequences of the primers were as follows: OCT4 forward, 5'-CTGGGTTGATCCTCGGACCT-3' and reverse, 5'-CCA 
TCGGAGTTGCTCTCCA-3'; survivin forward, 5'-AGGACC ACCGCATCTCTACAT-3' and reverse, 5'-AAGTCTGGC TCGTTCTCAGTG-3'; and GAPDH forward, 5'-GGAGCG AGATCCCTCCAAAAT-3' and reverse, 5'-GGCTGTTGT CATACTTCTCATGG-3'.

For miRNA detection, a PureLink miRNA isolation kit (Invitrogen; Thermo Fisher Scientific, Inc.) was used to isolate the miRNAs. Small RNAs (50 ng) were processed for poly-A addition and cDNA synthesis, as previously described. Quantitative real-time PCR was then conducted using a Bio-Rad CFX96 system with FAM/FITC to determine the expression level of the miRNAs of interest. Expression levels were normalized against U6 small nuclear RNA.

Western blotting. Cells were washed with PBS and lysed in an RIPA buffer (Sigma-Aldrich; Merck KGaA). Protein concentration was determined using the bicinchoninic acid (BCA) method of protein determination with a Microplate Manager V6 (Bio-Rad Laboratories, Inc.). Proteins (20 $\mu \mathrm{g})$ were separated on $8-10 \%$ SDS-PAGE gel and then transferred to PVDF membranes (EMD Millipore). Membranes were blocked in 5\% non-fat milk in PBST for $1 \mathrm{~h}$ at room temperature, and then incubated with diluted primary antibodies against GAPDH (dilution 1:1,000; cat. no. sc-166574; Santa Cruz Biotechnology, Inc.), TR4 (dilution 1:500; product code ab109513; Abcam), OCT4 (dilution 1:500; product no. 2750; Cell Signaling Technology, Inc.) overnight at $4^{\circ} \mathrm{C}$. The blots were then incubated with HRP-conjugated secondary antibody (goat anti mouse; 1:10,000; cat. no. A16078; or goat anti rabbit; 1:10,000; cat. no. A16110; both from Thermo Fisher Scientific, Inc.) for $1 \mathrm{~h}$ at room temperature, washed, and developed in an ECL system (Bio-Rad Laboratories, Inc.).

MTT assay. Cells were infected with lentivirus for $48 \mathrm{~h}$ and then seeded in a 96-well plate at a concentration of $1 \times 10^{4}$ cells/well. Docetaxel was added at various concentrations $(0,2,5,10$, $15,20,30,40 \mu \mathrm{g} / \mathrm{ml}$ ) to the wells $24 \mathrm{~h}$ later. After $48 \mathrm{~h}$ of incubation, cells from each well were treated with MTT $(0.5 \mathrm{mg} / \mathrm{ml})$ for $4 \mathrm{~h}$ at $37^{\circ} \mathrm{C}$. The absorbance at $570 \mathrm{~nm}$ was determined using a microplate reader (Model 550; Bio-Rad Laboratories, Inc.).

Chromatin immunoprecipitation assay. Regarding chromatin immunoprecipitation (ChIP), cultured C4-2 prostate cancer cells $\left(2 \times 10^{7}\right)$ were harvested and treated with $1 \%$ formaldehyde at room temperature for $10 \mathrm{~min}$ to cross-linked DNA. After sonication, cross-linked chromatin was precleared with A/G-agarose protein beads and then immunoprecipitated using anti-TR4-specific antibody (product code ab109513; Abcam) or Anti-Histone H3 (acetyl K27) antibody (product code ab4729) or IgG (product code ab172730) overnight at $4^{\circ} \mathrm{C}$. $\operatorname{IgG}$ was used as the negative control and Anti-Histone H3 as the positive control. Supernatants from the no-antibody-added samples were used to measure total input chromatin. The chromatins were incubated overnight at $65^{\circ} \mathrm{C}$ to reverse cross-links. DNA was then treated with $20 \mu \mathrm{g} / \mathrm{ml}$ RNase A $\left(37^{\circ} \mathrm{C}, 30 \mathrm{~min}\right)$, purified using gel extraction columns (OMEGA), and resuspended in $50 \mathrm{ml}$ TE buffer. Then, the TR4 occupancy on chromatin was assessed by PCR with locus-specific primers.
Luciferase reporter assay. The full-length sequence of the human miR-145 promoter was cloned into a pGL3-basic luciferase reporter vector (Promega Corporation). Site-directed mutagenesis of the TR4 binding site in the promoter was achieved with the Quick-Change mutagenesis (Stratagene; Agilent Technologies, Inc.) according to the manufacturer's protocols.

The 3'UTR of the human OCT4 gene with predicted miRNA-responsive elements was cloned into the pGL3-promoter vector (Promega Corporation) downstream of the firefly luciferase ORF. Site-directed mutagenesis of miRNA-responsive elements in 3'UTR was achieved with the Quick-Change mutagenesis according to the manufacturer's protocols.

Cells were placed in 24-well plates. The plasmids or chemical miRNA mimics were transfected with Lipofectamine 3000 (Thermo Fisher Scientific, Inc.) according to the manufacturer's instructions. PRL-TK was used as an internal control. Cell lysates were prepared with Passive Lysis Buffer (Promega Corporation) $24 \mathrm{~h}$ after transfection. Luciferase activity was measured using the Dual-Luciferase Reporter Assay (Promega Corporation) according to the manufacturer's manual. Firefly luciferase activity was normalized by comparing to Renilla luciferase activity.

In vivo mouse model. Animal experiments were performed according to a protocol approved by the Ethics Committee of the Second Affiliated Hospital of Soochow University (Suzhou, China) and followed the ARRIVE Guidelines Checklist (27). Forty 8 -week-old BALB/c-Foxn $1^{\text {nu }}$ male mice with a body weight of 18-19 g were purchased from the Model Animal Research Center of Nanjing University (Nanjing, China). They were housed under standard conditions with a 12-h light/dark cycle at $23 \pm 3^{\circ} \mathrm{C}, 55 \pm 5 \%$ relative humidity, and access to food and water ad libitum. All the mice received anesthesia by intraperitoneal injection of pentobarbital $50 \mathrm{mg} / \mathrm{kg}$. Then PC3 human PCa cells $\left(5 \times 10^{6} /\right.$ mouse) mixed with Matrigel (BD Biosciences) were concurrently injected into the right axillary space of each mouse. Tumor sizes were measured every four days with a caliper. The mice were randomly divided into four groups 21 days after tumor cell inoculation, specifically, a negative control group (NC), shTR4 group (shTR4), shmiR-145 group (sh145), and a shTR4+shmiR-145 (shTR4+145) group. Lentivirus was injected into the tumor of each mouse. After 48 h, 5 mg/kg docetaxel (Sigma Aldrich; Merck KGaA) was injected intraperitoneally twice a week for three weeks. Then the mice were sacrificed by cervical dislocation and tumors were removed for further characterization.

The duration of the experiment was 51 days. During the experiment, mice health and behaviour were monitored daily. The humane endpoint for mice was a tumor size $>17 \mathrm{~mm}$ in diameter of any mouse. All 40 mice were euthanized by cervical dislocation. No mouse was found dead before euthanasia. Animal welfare considerations were taken including anaesthetics (intraperitoneal injection of pentobarbital $50 \mathrm{mg} / \mathrm{kg}$ ) to minimize suffering and distress.

Immunohistochemistry $(I H C)$. Tumor tissues were fixed in $4 \%$ neutral buffered paraformaldehyde (room temperature, $12 \mathrm{~h}$ ) and embedded in paraffin. Immunohistochemical staining was performed on $4-\mu \mathrm{m}$ sections of paraffin blocks. After blocking 


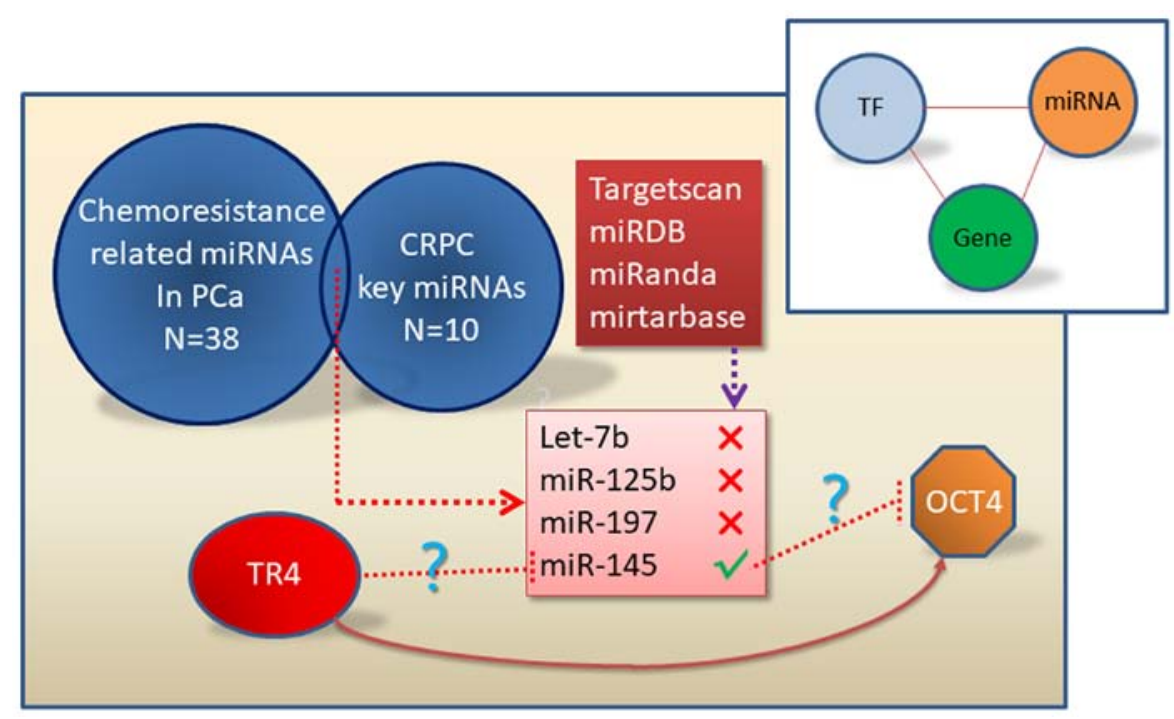

Figure 1. miR-145 is predicted to join the TR4/OCT4 signaling to mediate PCa chemoresistance. A total of 38 chemoresistance-related miRNAs in PCa were listed by text mining. The intersection between the 38 miRNAs and the 10 key miRNAs in CRPC previously identified by our research group was calculated. Four miRNAs (let-7b, miR-125b, miR-197, and miR-145) were revealed to play key roles in the chemoresistance in CRPC. Four online databases (TargetScan, miRDB, miRanda, miRTarBase) were used and predicted miR-145 as the only miRNA to target OCT4. TR4, testicular nuclear receptor 4; OCT4, octamer-binding transcription factor 4; PCa, prostate cancer; miR/miRNAs, microRNAs; CRPC, castration-resistant prostate cancer.

with $3 \%$ hydrogen peroxide for $10 \mathrm{~min}$ at room temperature, the slides were then incubated overnight at $4^{\circ} \mathrm{C}$ with primary antibody. The primary antibodies, OCT4 (diluted 1:800; product no. 2750; Cell Signaling Technology, Inc.) and survivin (diluted 1:500; product code ab76424; Abcam) were used for staining. The slides were then incubated with the biotinylated goat-anti-rabbit secondary antibody (diluted 1:500; product no. BA-1000; Vector Laboratories, Inc.) for $30 \mathrm{~min}$ at room temperature, and visualized by VECTASTAIN ABC peroxidase system and peroxidase substrate DAB kit (Vector Laboratories, Inc.) according to the manufacturer's instructions. The slides were analyzed using an Olympus BX51 fluorescence microscope (Olympus Corporation).

Statistical analysis. Values were expressed as the mean \pm standard deviation. The unpaired t-test was used for comparing two groups and ANOVA followed by the Tukey's test was used for multiple comparisons using SPSS 19.0 software (IBM Corp.). $\mathrm{P}<0.05$ (two-sided) indicated a statistically significant difference.

\section{Results}

miR-145 mediates PCa chemoresistance. In our previous study, it was revealed that TR4 can mediate the chemoresistance of PCa by upregulating the expression of OCT4 at the transcriptional level (15). However, more evidence revealed that miRNAs and TFs may cooperate to control gene expression via TF-miRNA-target axes. For example, TR4 has been revealed to promote prostate cancer metastasis through the TR4/miR-373/TGF $\beta$ R2 signaling axis (28). This prompted us to investigate the existence of a TR4/miRNA/OCT4 axis that regulates chemoresistance of $\mathrm{PCa}$.

In total, 38 miRNAs that are related to chemoresistance in PCa by text mining were collected. The intersection was calculated between these 38 miRNAs and another ten key
miRNAs in CRPC previously identified (14) since chemoresistance occurs in the CRPC stage. It was revealed that four miRNAs, mainly let-7b, miR-125b, miR-197 and miR-145 played essential roles in the chemoresistance of CRPC (Fig. 1). Then, four online databases (TargetScan, miRDB, miRanda, miRTarBase) were used to predict whether any of these four miRNAs can target OCT4. Unfortunately, only miR-145 was predicted to target OCT4 in one database (miRTarBase). Consequently, miR-145 was the only miRNA needed for further validatation (Fig. 1).

TR4 suppresses miR-145 but promotes OCT4 expression in $P C a$ cell lines. It was first evaluated whether TR4 can affect the expression of miR-145. PCa PC3 cells were infected with either a TR4 knockdown (shTR4) or negative control (scr) lentivirus, and then the miR-145 expression levels were determined by qPCR. OCT4 mRNA and protein expression levels were also determined by qPCR and western blot analyses. As revealed in Fig. 2A, the knocking down of TR4 significantly increased the expression of miR-145 while decreasing OCT4 mRNA and protein expression in PC3 cells.

Similarly, the other two PCa cell lines, C4-2, and DU-145, were used for validatation. As anticipated, upon inhibition of TR4, miR-145 increased and OCT4 decreased in both C4-2 (Fig. 2B) and DU-145 cells (Fig. 2C).

To further confirm the TR4 negative regulation on miR-145, TR4 was overexpressed in all three cell lines using lentivirus. The results revealed that miR-145 expression decreased and OCT4 increased at both mRNA and protein levels (Fig. 2D-F).

The results (Fig. 2A-F) revealed that TR4 suppressed miR-145 but promoted OCT4 expression in PCa.

miR-145 negatively regulates OCT4 expression in PCa cell lines. Since the TR4/miR-145 regulation was confirmed, the following goal was to assess the miR-145/OCT4 signal. 
A a

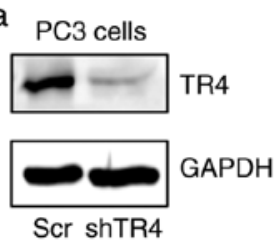

B a

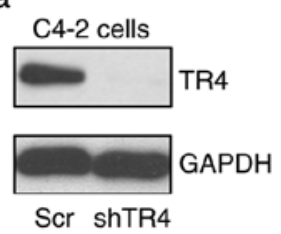

C a

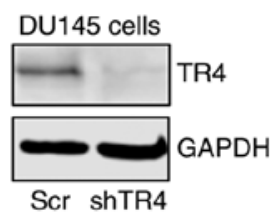

D a

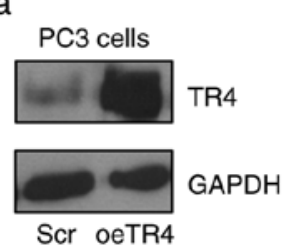

E a

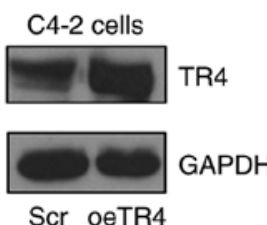

F a

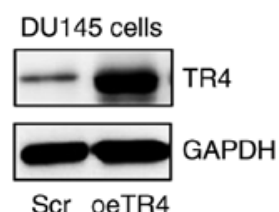

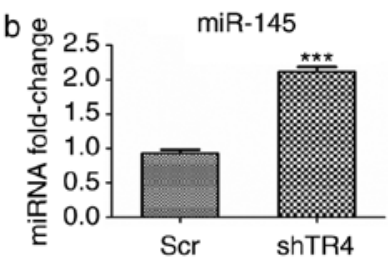

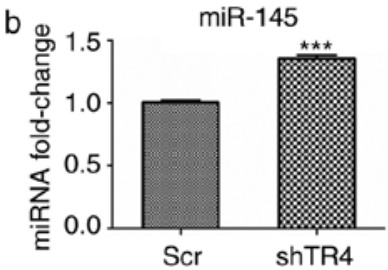

b g 2.0 miR-145
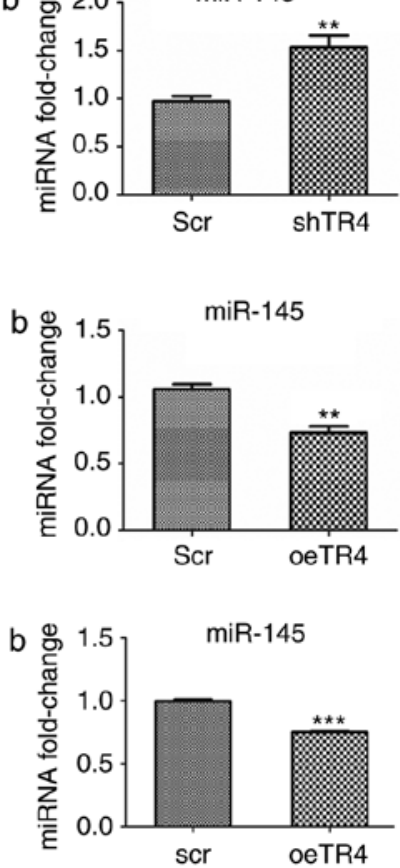

b

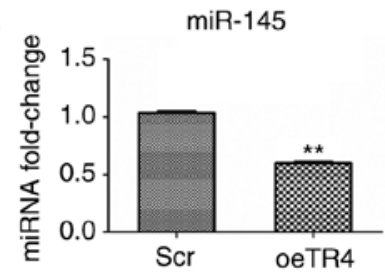

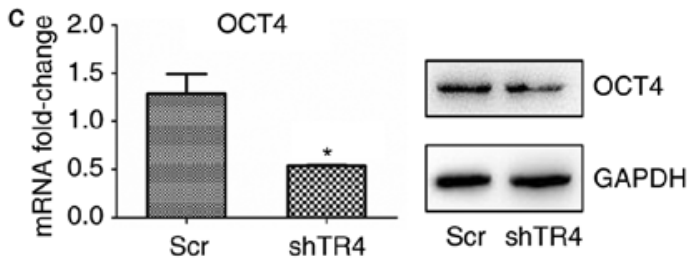
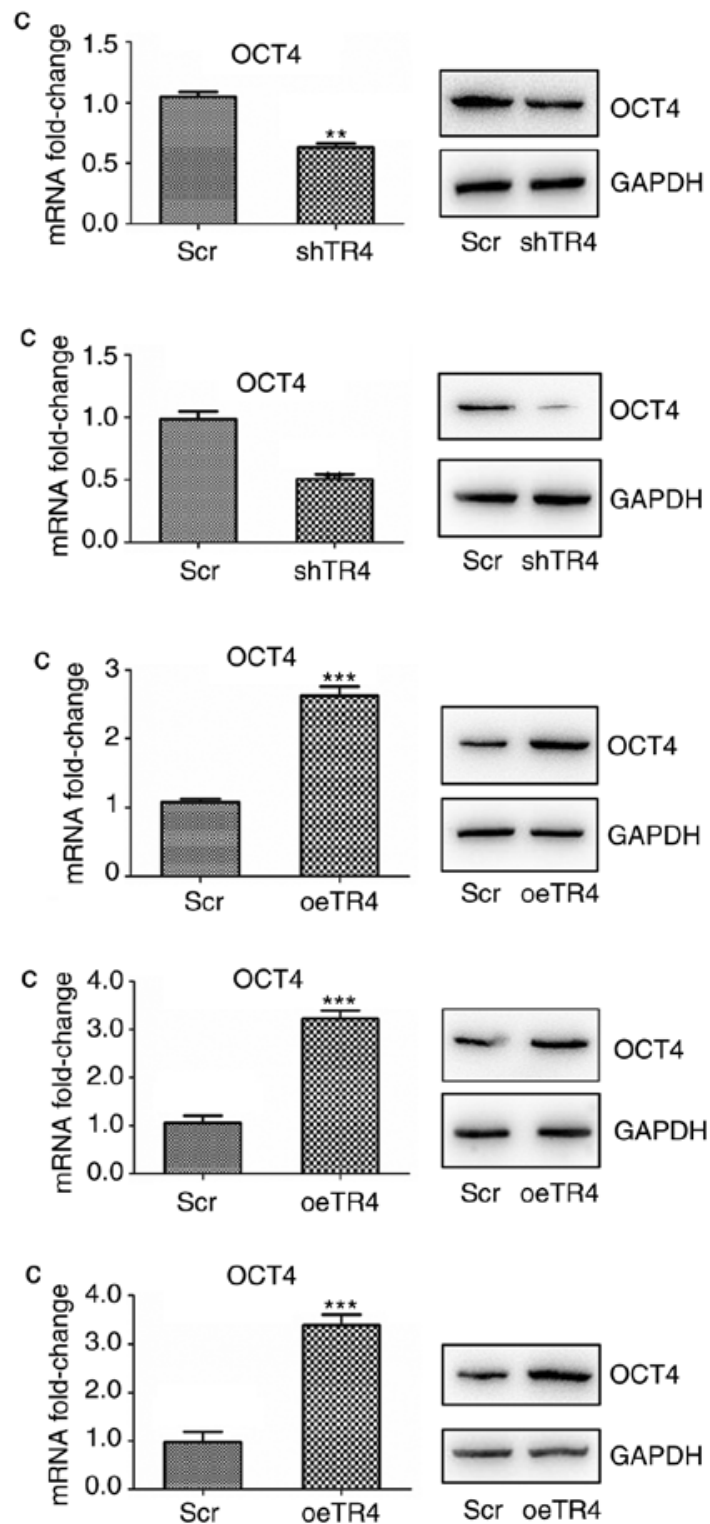

Figure 2. TR4 suppresses miR-145 but promotes OCT4 expression in PCa cell lines. TR4 was (A-a to C-a) inhibited or (D-a to F-a) overexpressed in three PCa cell lines (PC3, C4-2, DU145). (A-b to F-b) The expression of miR-145 was determined by qPCR. (A-c to F-c) OCT4 mRNA and protein expression levels were determined by qPCR and western blotting analyses, respectively. ${ }^{*} \mathrm{P}<0.05,{ }^{* *} \mathrm{P}<0.01$ and ${ }^{* * *} \mathrm{P}<0.001$. TR4, testicular nuclear receptor 4 ; miR, microRNA; OCT4, octamer-binding transcription factor 4; PCa, prostate cancer; qPCR, quantitative PCR; scr, scambled; oe, overexpressed.

miR-145 has been reported to directly target OCT4 in several cancer types $(29,30)$. However, in PCa, the miR-145/OCT4 signal was reported only once in one cell line (PC3), and the detailed mechanism was not discussed (31). Consequently, it was neccessary to confirm the effect of miR-145 on OCT4 expression in more PCa cell lines.

Experiments were performed on the PC3 PCa cell line and chemically synthesized miR-145 mimic or negative control was transfected into PC3 cells. OCT4 expression was evaluated using qPCR and western blotting. As revealed in Fig. 3A, when miR-145 was overexpressed, OCT4 expression decreased significantly both at the mRNA and protein levels.

In the other two cell lines, C4-2 and DU-145, miR-145 mimics were transfected and similar results were obtained (Fig. 3B and C).

For further confirmation, the miR-145 inhibitor was transfected into the three cell lines. The results revealed that the expression of OCT4 significantly increased in all three cell lines at the mRNA and protein level when miR-145 was inhibited (Fig. 3D-F). 

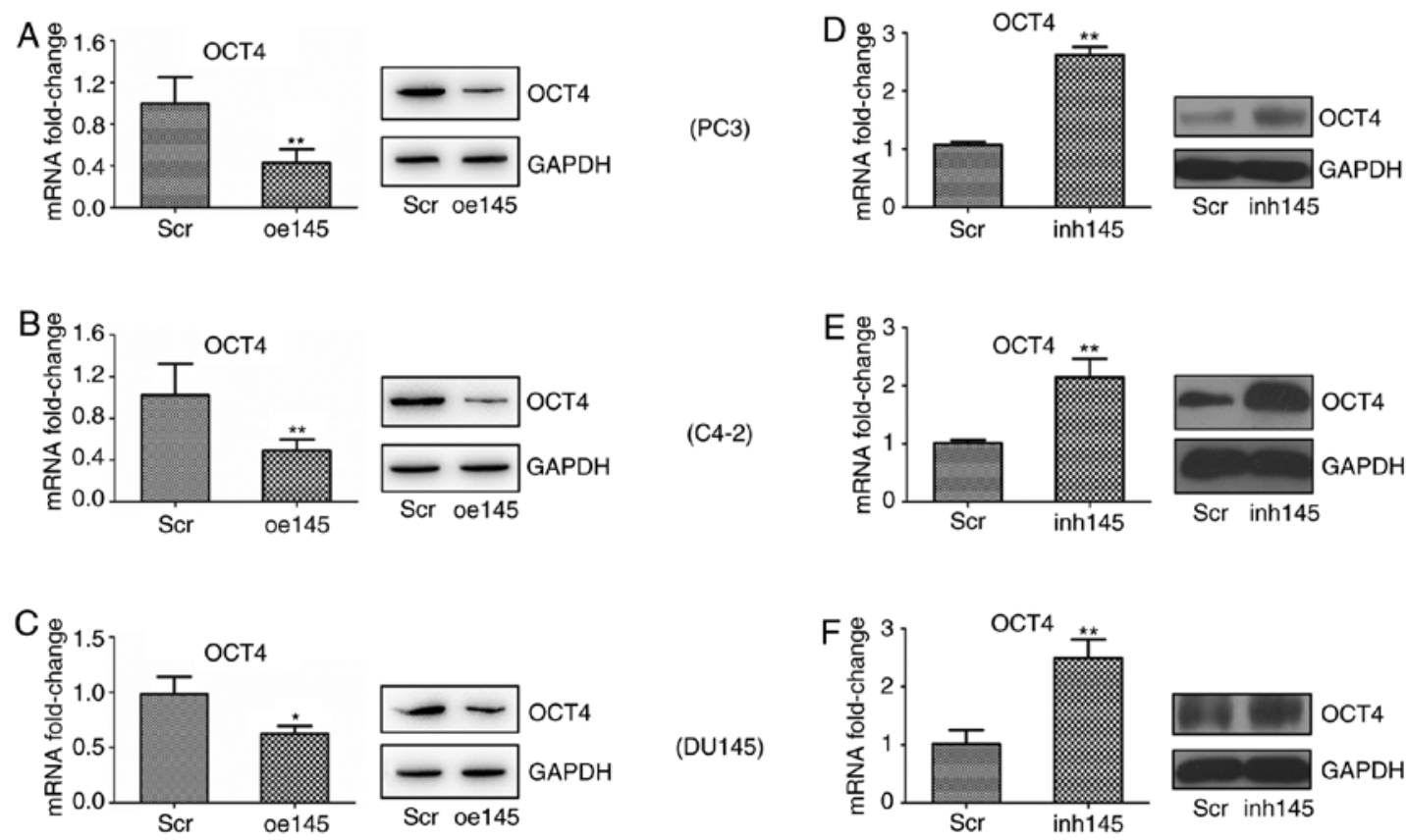

Figure 3. miR-145 negatively regulates OCT4 expression in PCa cell lines. miR-145 was (A-C) overexpressed or (D-F) inhibited in three PCa cell lines (PC3, C4-2, DU145). OCT4 mRNA and protein expression levels were determined by qPCR and western blotting. " $\mathrm{P}<0.05$ and ${ }^{* *} \mathrm{P}<0.01$. miR, microRNA; OCT4, octamer-binding transcription factor 4; PCa, prostate cancer; qPCR, quantitative PCR; scr, scambled; oe, overexpressed; inh, inhibitor.
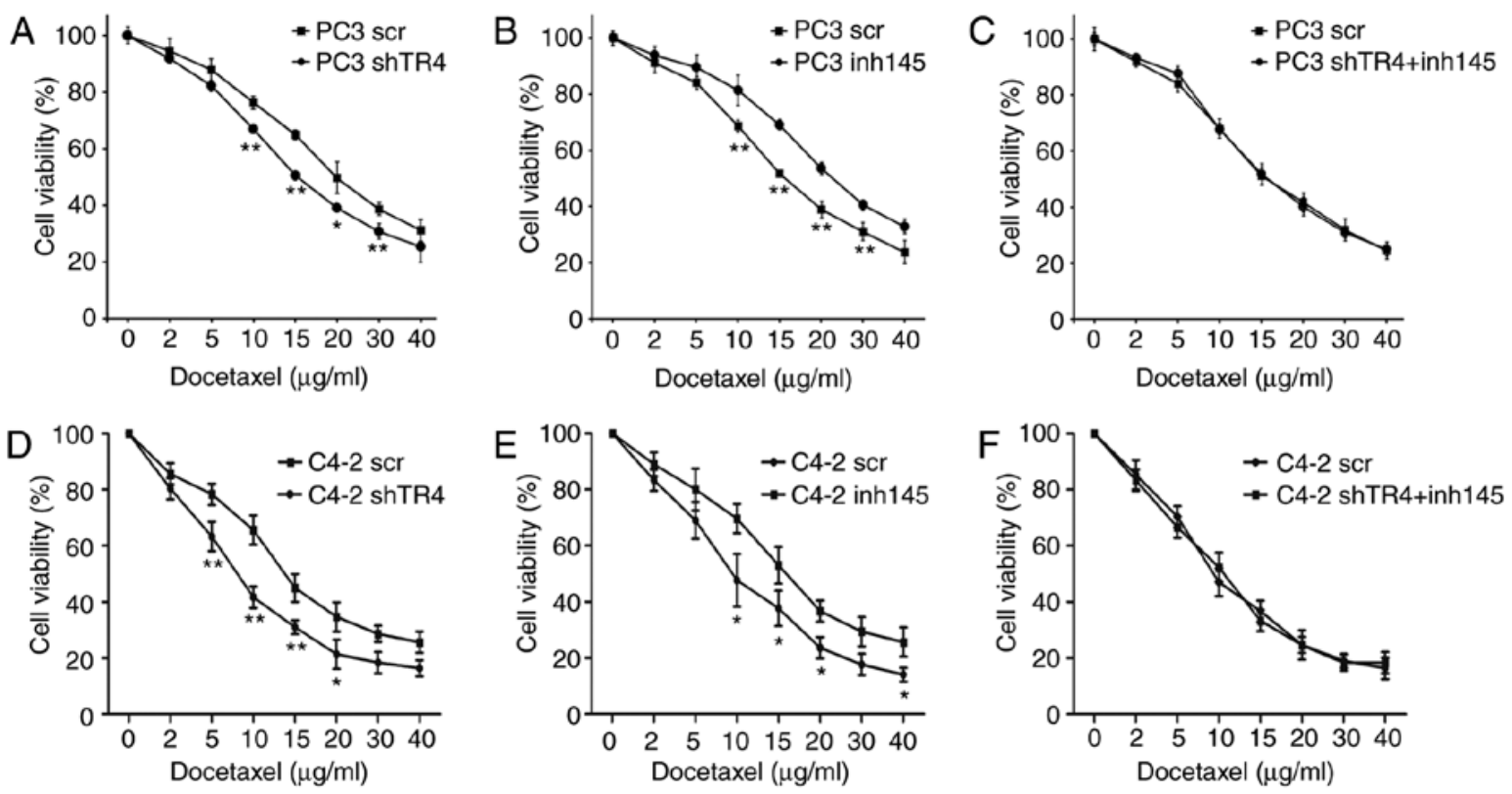

Figure 4. TR4-mediated chemoresistance in PCa is attenuated by miR-145. PC3 or C4-2 cells were divided into three groups: (A and D) TR4-silenced group, (B and E) miR-145 inhibitor group, and (C and F) TR4 and miR-145 double-inhibition group. Cells were treated with a specific concentration $(0,2,5,10,15,20,30,40 \mu \mathrm{g} / \mathrm{ml})$ of docetaxel. Cell survival after the treatment of docetaxel was recorded. ${ }^{*} \mathrm{P}<0.05$ and ${ }^{* *} \mathrm{P}<0.01$. TR4, testicular nuclear receptor 4; PCa, prostate cancer; miR, microRNA; scr, scrambled; sh, short hairpin; inh, inhibitor.

Collectively, the results (Fig. 3A-F) revealed that miR-145 suppressed OCT4 expression in PCa cells.

TR4-mediated chemoresistance in PCa is attenuated by $m i R-145$. Although all of the data revealed the regulatory axis of the TR4/miR-145/OCT4 signal, further experimention was conducted to confirm that miR-145 had a real function in TR4/OCT4-mediated PCa chemoresistance.
First, TR4 expression was inhibited using a lentivirus in PC 3 cells and the cell survival was assessed after treatment with docetaxel. The results revealed that the sensitivity to docetaxel of the shTR4 group was significantly increased compared with the control group (Fig. 4A). Then, miR-145 was inhibited using a lentivirus and it was reveled that PC3 cells lacking miR-145 were more resistant to docetaxel compared with the control group (Fig. 4B). Next, the 
A

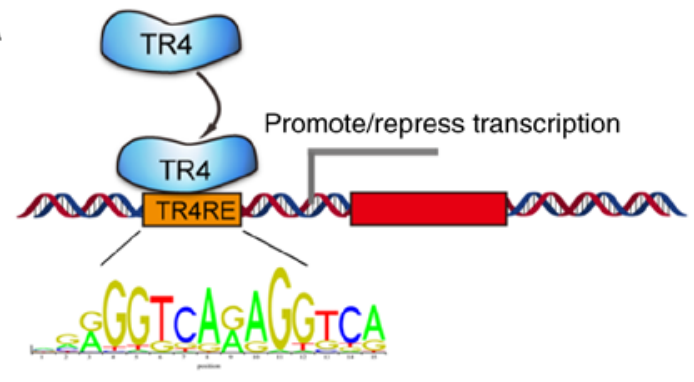

B miR-145 promoter

AGGCT I CCT T CCT CCI ACC CCCT T C CCCCAAT CT T AT T CAT

-a442 |111111111111111111111111111111111111

I CCGAAGGAAGGAGG ATGGGGGAGGGGGT TAGAAT AAGT A

TR4RE1 (-427 -413)

TR4RE2 $(-349 \sim-335)$

I AAT T AAAGAAGGCC AAGGCI CAGGGGITAAGT GACII ACI

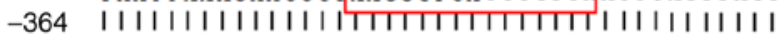
AT T AAT T T CT T C CG GI I C CGAG T C CCAAT T CACT GAAT GA TR4RE3 (-220 -206)

ACACATGAGCCGTGCAGACAGCAGAGGGCAGT CCTGGGGG

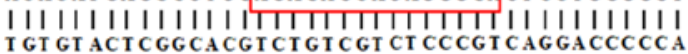
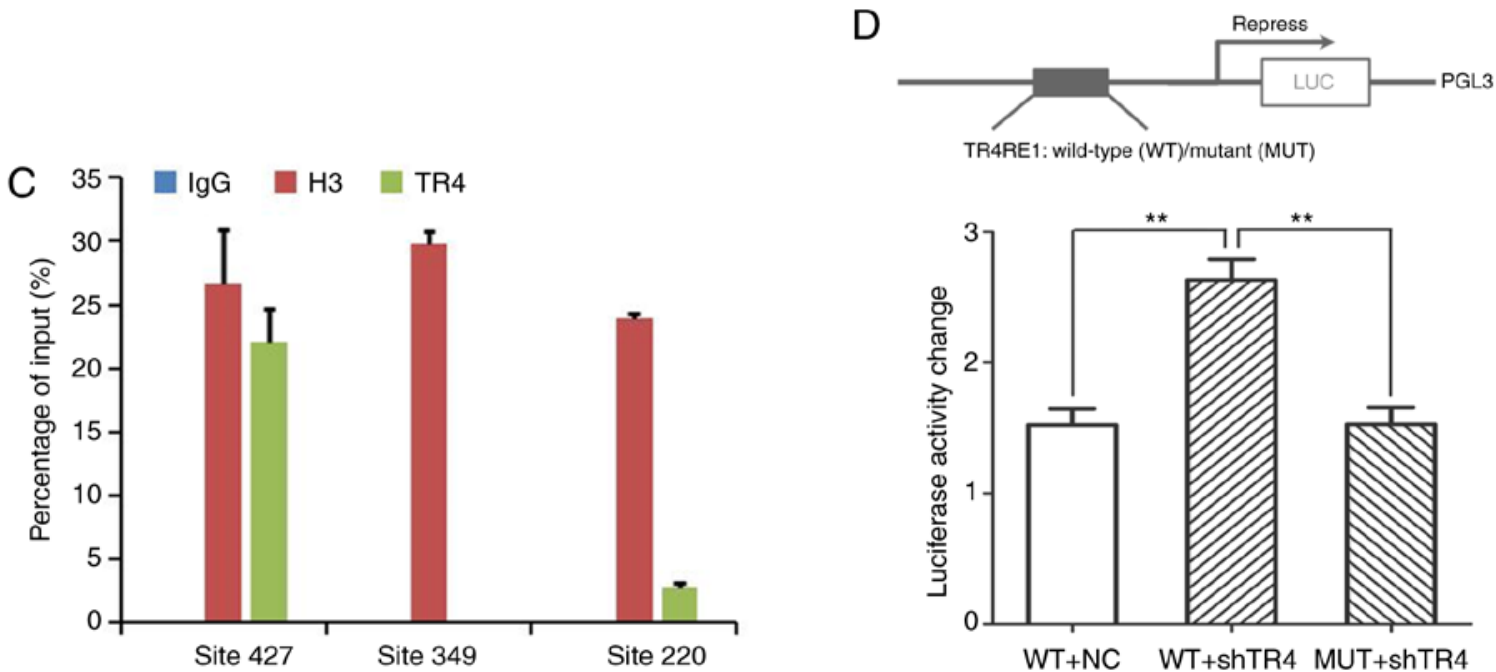

Figure 5. TR4 inhibits the expression of miR-145 at the transcriptional level. (A) TR4RE motif sequences. (B) Three putative TR4REs predicted by JASPAR from the miR-145 promoter. (C) ChIP assay revealed TR4 protein binding to the chromatin at the first putative TR4RE in the miR-145 promoter. (D) Promoter luciferase reporter assay revealed that TR4 could inhibit miR-145 wild-type promoter activity but had no effect on the mutant promoter. TR4, testicular nuclear receptor 4; miR, microRNA; TR4RE, TR4 response element; WT, wild-type; MUT, mutant; NC, negative control; sh, short hairpin. ${ }^{* * *} \mathrm{P}<0.01$.

chemosensitivity to docetaxel between the control group and the TR4/miR-145 double-knockdown group was compared; the results revealed that there was no significant difference in chemosensitivity to docetaxel between these two groups (Fig. 4C).

One more cell line (C4-2 line) was used to repeat the three tests. As anticipated, TR4 shRNA increased C4-2 sensitivity to docetaxel (Fig. 4D), while the inhibition of miR-145 caused more chemoresistance to C4-2 cells (Fig. 4E). Notably, in C4-2 cells, TR4/miR-145 double-knockdown cells also had a similar sensitivity to docetaxel when compared to the control group (Fig. 4F).

The results (Fig. 4A-F) revealed that miR-145 could attenuate TR4 mediated chemoresistance in PCa.

TR4 inhibits the expression of miR-145 at the transcriptional level. TR4 is a transcription factor that can regulate a large number of molecules in $\mathrm{PCa}$ and other diseases at the transcriptional level (25) (Fig. 5A). Given this reason, it was decided to further investigate whether TR4 could bind to the promoter region of miR-145 to regulate it.

The online tool JASPAR was used to predict the putative TR4 response elements (TR4REs) for the promoter of miR-145. As revealed in Fig. 5B, three putative TR4REs were identified, including TR4RE1 ( -427 to -413), TR4RE2 ( -349 to -335$)$, and TR4RE3 ( -220 to -206).
Then, a ChIP assay was performed to verify whether TR4 binds to any of the three putative TR4REs. As revealed in Fig. 5C, IgG served as the negative control and anti-histone $\mathrm{H} 3$ as the positive control; the TR4 protein could only robustly bind to the first predicted TR4 response element (TR4RE1, $\sim-427$ to -413 ).

Then, the full promoter DNA sequence of miR-145 was cloned into the PGL-3 primary luciferase vector and another promoter with a mutant in the TR4RE1 region. The luciferase assays were then performed (Fig. 5D); upon the inhibition of TR4, the activity of miR-145 promoter significantly increased compared with the control group. As anticipated, when the wild-type promoter was substituted with the mutant promoter, TR4 inhibition no longer had any effect on luciferase activity.

Collectively, the results (Fig. 5A-D) revealed that TR4 transcriptionally supressed miR-145 via binding to the TR4 response element ( - 427 to -413$)$ in the promoter region of miR-145.

miR-145 targets OCT4 by directly targeting its 3'untranslated $\left(3^{\prime} U T R\right)$ region. The mechanism for the regulation of miR-145 on OCT4 has been previously reported in other cancers but not in $\mathrm{PCa}(29,30)$. To further confirm the present findings, it was attempted to reveal the mechanism in C4-2 PCa cells.

As revealed in Fig. 6A, the 3'UTR of OCT4 was predicted to have putative miRNA response elements (MRE) of miR-145 

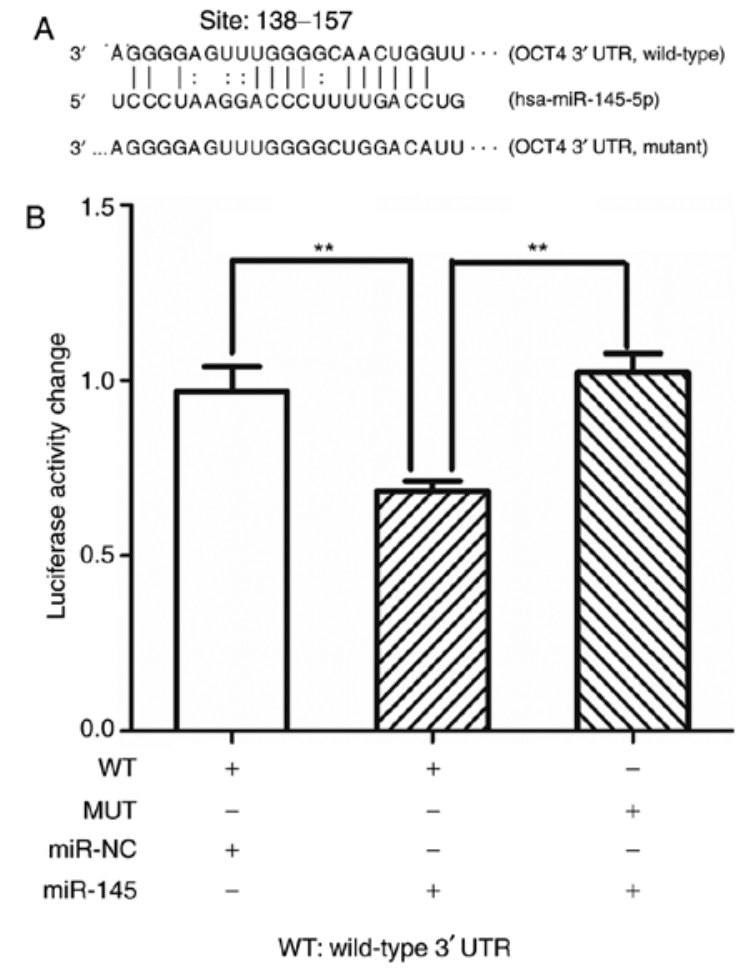

MUT: mutant $3^{\prime}$ UTR

Figure 6. miR-145 targets OCT4 by directly targeting its 3'UTR. (A) The 3'UTR of OCT4 is predicted to have putative MRE of miR-145 (site: $\sim 138-157$ ). (B) The full 3'UTR sequence of OCT4 was cloned into the PGL-3 promoter-luciferase vector downstream to the firefly luciferase. Upon transfection of miR-145, luciferase activity decreased significantly. miR-145 did not affect the mutated 3'UTR. .* $\mathrm{P}<0.01$. miR, microRNA; OCT4 octamer-binding transcription factor 4; 3'UTR, 3'untranslated region; MRE, miRNA response elements; WT, wild-type; MUT, mutant; NC, negative control.

(site: 138-157). Thus, the full 3'UTR sequence of OCT4 was cloned into the PGL-3 promoter-luciferase vector downstream to the firefly luciferase. In addition, the 3'UTR was cloned with a mutant in the seed region. Luciferase assays were then performed, and as revealed in Fig. 6B, upon transfection of miR-145, luciferase activity significantly decreased compared with the control group. As anticipated, when the wild-type 3'UTR was substituted with the mutant one, miR-145 no longer had any effect on luciferase activity.

Collectively, the results (Fig. 6A and B) revealed that miR-145 targeted OCT4 by directly targeting its 3'UTR.

TR4 causes chemoresistance to PCa via miR-145/OCT4 signals in vivo. As the in vitro data revealed that TR4 regulates the chemosensitivity of PCa via the miR-145/OCT4 signal, the present study investigated whether the same effects were observed in vivo.

A total of 40 male nude mice were used in the experiment. For each mouse, $5 \times 10^{6}$ cells were subcutaneously injected into the right axillary space. The mice were then divided into four groups 21 days after tumor cell inoculation: A control group (NC), a TR4-inhibiting group (shTR4), a miR-145-inhibiting group (sh145), and a TR4/miR-145 double-inhibiting group (shTR4+145). Lentivirus was injected into the tumor of each mouse. After $48 \mathrm{~h}, 5 \mathrm{mg} / \mathrm{kg}$ docetaxel was injected intraperitoneally at a frequency of twice a week for 3 weeks. Tumor sizes were measured every 4 days and the mice were then sacrificed. Then, the tumors were collected and the tumor volumes were calculated.

The results demonstrated that tumors in the shTR4 group were smaller than those in the control group (Fig. 7A-C). Tumors in the sh145 group were had a larger tumor size than those in the control group (Fig. 7A-C). Notably, tumors in the TR4/miR-145 double-inhibiting group had a similar size compared to those in the control group (Fig. 7A-C).

The expression of miR-145, OCT4, and survivin was then determined using qPCR. Survivin is the downstream gene of OCT4 (32) and is an antiapoptotic factor in PCa (33). As revealed in Fig. 7D-F, miR-145 was increased while OCT4 and survivin were decreased in the shTR4 group. In the sh145 group, OCT4 (Fig. 7E) and survivin (Fig. 7F) mRNA were significantly increased. Notably, the shTR4 effect on OCT4 and survivin was attenuated by concurrent inhibition of miR-145 (shTR4+145 group compared with shTR4 group) (Fig. 7E and F).

Similarly, when immunohistochemical staining was used to analyze OCT4 and survivin protein expression, it was revealed that the same expression pattern of both OCT4 (Fig. 7G) and survivin (Fig. $7 \mathrm{H}$ ) occurred in the four groups as compared with the qPCR results.

Collectively, these data indicated that TR4 transcriptionally inhibited the expression of miR-145 and consequently promoted the expression of OCT4, causing chemoresistance of $\mathrm{PCa}$ in vitro and in vivo. miR-145 could reverse the TR4 effect on chemosensitivity in $\mathrm{PCa}$. The in vitro and in vivo data obtained in the present study confirmed the TR4/miR-145/OCT4 signaling in PCa chemoresistance.

\section{Discussion}

The mechanism by which CRPC patients display different therapeutic efficacy to docetaxel needs to be further explored. In our previous study, it was identified that TR4 may promote chemoresistance of $\mathrm{PCa}$ via increasing the expression of OCT4 (15). In the present study, the purpose was to identify an 'intermediary miRNA' between TR4 and OCT4 to regulate the chemosensitivity in PCa. miR-145 was predicted as the key miRNA by using integrative bioinformatics analysis. Findings revealed that TR4 could inhibit miR-145 expression while increasing OCT4 expression and, consequently, causing chemoresistance to docetaxel in vitro and in vivo and miR-145 was able to reverse the effects. Furthermore, findings demonstrated that TR4 enhanced the chemoresistance of $\mathrm{PCa}$ at least partially via the axis of TR4/miR-145/OCT4.

The role of nuclear receptors in prostate cancer has received increasing attention. For example, recent study has revealed that ROR $\gamma$ mediated resistance of prostate cancer to doxorubicin chemotherapy (34). Nuclear receptor HNF4 $\alpha$ funtioned as a tumor suppressor in prostate cancer via its induction of p21-driven cellular senescence (35). In addition, TR4 is a nuclear receptor and has been revealed to play an important role in tumors, especially prostate cancer (28).

TR4 nuclear receptor plays a crucial role in the development and progression of various types of cancer including liver (36), breast (37), lung (38), and renal cancer (39). For example, TR4 

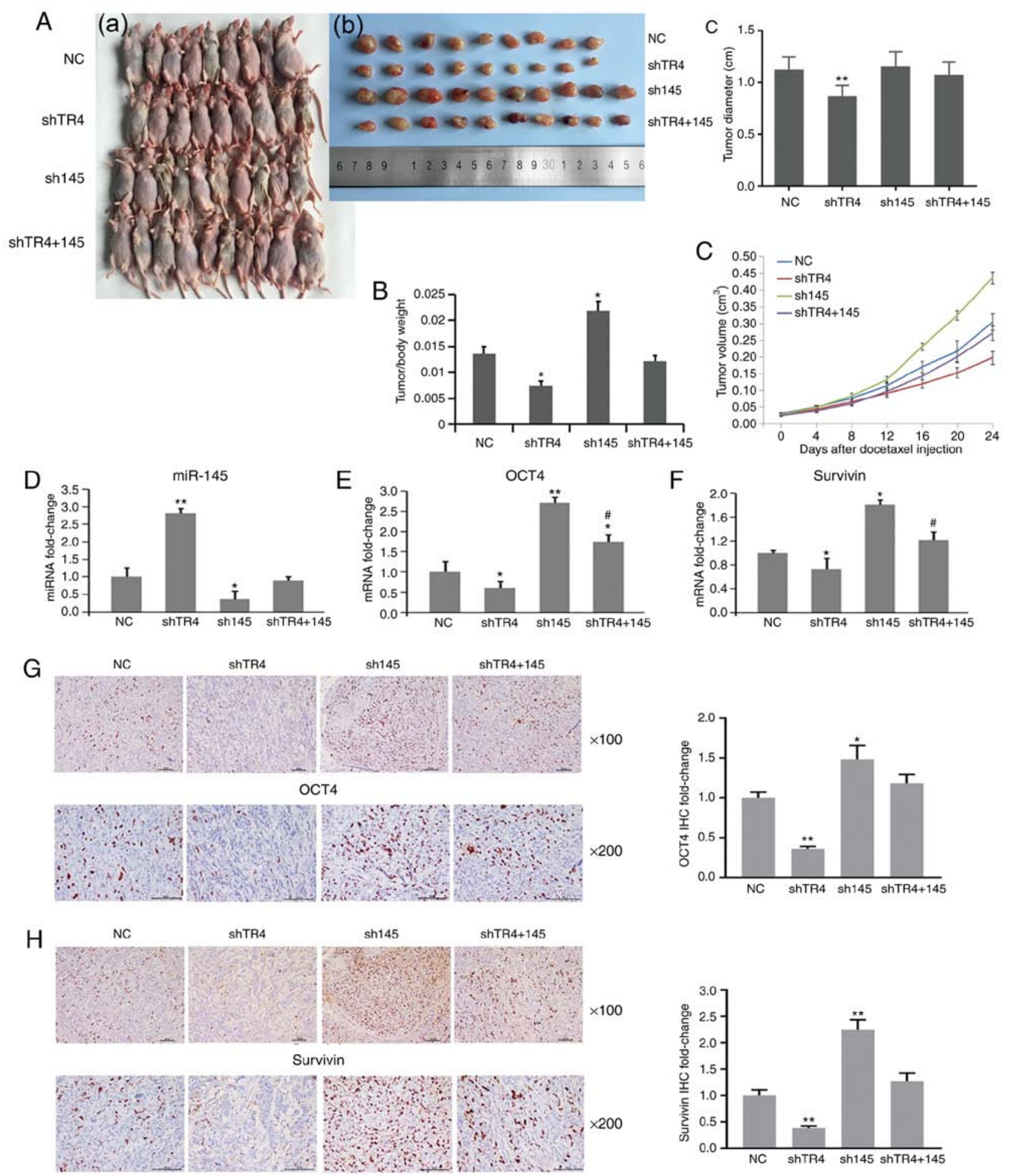

Figure 7. TR4 causes chemoresistance to PCa via miR-145/OCT4 signals in vivo. Forty male nude mice were used in the experiment. For each mouse, $5 \times 10^{6}$ cells were subcutaneously injected into the right axillary space. Then the mice were divided into four groups 21 days after tumor cell inoculation: The control group (NC), the TR4-inhibiting group (shTR4), the miR-145-inhibiting group (sh145), and the TR4/miR-145 double-inhibiting group (shTR4 and 145). Lentivirus was injected into the tumor of each mouse. After $48 \mathrm{~h}, 5 \mathrm{mg} / \mathrm{kg}$ docetaxel was injected intraperitoneally at a frequency of twice a week for three weeks. Mice were sacrificed and (A-a to A-c) tumors were collected, and (B) tumor/body weight ratio was recorded. (C) The tumor volume was measured every four days after docetaxel injection. Expression of (D) miR-145, (E) OCT4, and (F) survivin of tumor tissue from each group was determined by qPCR. Expression of $(\mathrm{G})$ OCT4 and $(\mathrm{H})$ survivin protein in tumor tissues from each group was determined by IHC staining. "P $<0.05$ and ${ }^{* * *} \mathrm{P}<0.01 .{ }^{*} \mathrm{P}<0.05$, shTR4+145 group compared with shTR4 group. TR4, testicular nuclear receptor 4; PCa, prostate cancer; miR, microRNA; OCT4, octamer-binding transcription factor 4; $\mathrm{NC}$, negative control; sh, short hairpin.

promoted miR-32-5p to suppress clear cell renal cell carcinoma metastasis via altering the miR-32-5p/TR4/HGF/Met signaling (40). Shen et al revealed that TR4 enhanced cisplatin chemosensitivity via altering ATF3 expression in hepatic cell carcinoma (36). TR4 is especially important in PCa because TR4 regulates $\mathrm{PCa}$ initiation and progression. TR4 has been 
revealed to inhibit tumor initiation (41) but promote metastasis (42), radiation resistance (43), and chemoresistance (44) via several distinct signals. High TR4 expression in $\mathrm{PCa}$ tissues was revealed to be correlated with high Gleason scores and is usually correlated with a poor prognosis (42). In the present study, it was revealed that TR4 could be the cause of chemoresistance to docetaxel in PCa. This result is consistent with a study by Chen et al (44) and our previous study, which revealed that TR4 promoted PCa stem cell-mediated chemoresistance (15). Thus, it was concluded by the findings in the present study that TR4 is a strong drive factor to promote $\mathrm{PCa}$ progression from various aspects of tumor behaviors.

Several studies have indicated that human miR-145 is involved in the progression of numerous cancers acting as a tumor suppressor (45-48). With regard to prostate cancer, miR-145 is expressed at a low level, therefore, the restoration of miR-145 could inhibit the proliferation and invasion restoring radiation sensitivity via targeting multiple genes $(49,50)$. Additionally, our previous study identified miR-145 as one of the key miRNAs involved in CRPC (14). However, no previous published data focused on the effect of miR-145 on the chemosensitivity of PCa. In the present study, it was revealed that miR-145 can re-sensitize PCa to docetaxel via targeting OCT4. The present findings provided more insight into the function of miR-145 in PCa.

Furthermore, the study linked the TR4 transcription factor and miR-145 together. Other transcription factors may also have this TF/miRNA regulation pattern. In lung adenocarcinoma, thyroid transcription factor- 1 has been reported to regulate miR-532-5p consequently inducing apoptosis (51). Nanog has been reported to transcriptionally suppress miR-200 family in colon cancer cells and induce epithelial-mesenchymal transition (52). ChIP-seq data also revealed that TR4 could bind to the upstream locus of multiple miRNAs (53). The existence of this TF/miRNA regulation can modulate the biological function in cells in a precised manner.

A previous study reported that miR-145 targeted AR and inhibited prostate cancer progression (54). In addition, AR could regulate the activity of OCT4 (55). The present study revealed that miR-145 could directly target the 3'UTR of OCT4 to regulate OCT4 in both AR-positive cell line C4-2 and AR-negative cell lines PC3 and DU145. Therefore, it was speculated that miR-145 may regulate OCT4 directly, as well as indirectly through the AR/OCT4 pathway, and thus the completion of the regulatory goal is ensured through multiple pathways.

There is increasing interest in the study of TF/miRNA/gene axes in different diseases. For instance, Qiu et al revealed that TR4 could promote PCa metastasis via TR4/miR-373/TGFßR2 signaling (28). Wang et al revealed that TR4 promoted clear cell renal cell carcinoma vasculogenic mimicry formation and metastasis via altering the TR4/miR490-3p/vimentin signal (39). All of these studies have added evidence to the TF/miRNA/gene axis hypothesis.

For further investigation, web-based TF/miRNA/gene prediction tools have been developed such as TransmiR (56). These prediction tools are constructed with specific algorithms and ChIP-Seq based data. However, numerous TFs are not included in the databases and ChIP-Seq data are obtained from a minimal number of cell lines, thus limiting the use of these database tools. In the present study, online TF/miRNA/gene prediction tools were not used; instead bioinformatics methods were used including text mining and traditional miRNA/mRNA interaction databases. The researchers consider that with the decreasing cost for ChIP-Seq, it is possible to perform more ChIP-seq experiments based on PCa cells to better study PCa.

The researchers admit that bioinformatics methods have limitations. As was observed, only one miRNA was identified in the present study indicating that some other relevant miRNAs may have been missed. In addition, the possibility of adapting this method to future studies is unknown yet.

The present study revealed that TR4 inhibited miR-145 promoting the chemoresistance to docetaxel. This prompted our research group to hypothesize that the expression of TR4 and miR-145 may be used in combination to decide whether individual patients could accept docetaxel treatment. On the other hand, TR4 is an orphan receptor that lacks ligands, thus it may not be easy to target. Theoretically, the researchers can treat PCa with miR-145, since small RNA-delivering techniques are approaching clinical use at present.

In conclusion, the present study associated the expression of the transcription factor TR4 to miR-145 in PCa. Ectopic TR4 inhibited miR-145, thus increasing the expression of OCT4 and consequently causing chemoresistance to docetaxel. These findings provide insight into the interplay between TR4 and miR-145 in PCa chemosensitivity and open up new perspectives concerning the function and regulatory axes of transcription factors.

\section{Acknowledgments}

Not applicable.

\section{Funding}

The present study was supported by the National Natural Science Foundation of China (grant nos. 81472776 and 81773221; to DY and JZ), the Natural Science Foundation of Jiangsu Province (grant nos. BK20161222 and BK20191170; to JZ and LX). It was also funded by Suzhou Science and Technology Planned Projects (grant nos. SYS201629, SS201857 and SYS2018062; to JZ and LX), and the Key Young Talents of Medicine in Jiangsu (grant no. QNRC2016875; to JZ)

\section{Availability of data and materials}

The researchers declare that the raw data, certificates of the cell lines and other materials can be provided, if requested.

\section{Authors' contributions}

JZ and PQ performed the experiments and statistical analyses, and created the figures. CC and GD assisted with the interpretation of data and reviewed the manuscript. JZ, LX and DY conceived the study and wrote the manuscript. All authors read and approved the manuscript and agree to be accountable for all aspects of the research in ensuring that the accuracy or integrity of any part of the work are appropriately investigated and resolved. 


\section{Ethics approval and consent to participate}

Animal experiments were performed according to a protocol approved by the Ethics Committee of the Second Affiliated Hospital of Soochow University (Suzhou, China) and followed the ARRIVE Guidelines Checklist.

\section{Patient consent for publication}

Not applicable.

\section{Competing interests}

The authors declare that they have no competing interests.

\section{References}

1. Cronin KA, Lake AJ, Scott S, Sherman RL, Noone AM, Howlader N, Henley SJ, Anderson RN, Firth AU, Ma J, et al: Annual report to the nation on the status of cancer, part I: National cancer statistics. Cancer 124: 2785-2800, 2018.

2. Chen W, Zheng R, Baade PD, Zhang S, Zeng H, Bray F, Jemal A, $\mathrm{Yu}$ XQ and He J: Cancer statistics in China, 2015. CA Cancer J Clin 66: 115-132, 2016.

3. Zhao F, Shen J, Yuan Z, Yu X, Jiang P, Zhong B, Xiang J, Ren G, $\mathrm{Xie} \mathrm{L}$ and Yan S: Trends in treatment for prostate cancer in china: Preliminary patterns of care study in a single institution. J Cancer 9: 1797-1803, 2018.

4. Huang Y, Jiang X, Liang X and Jiang G: Molecular and cellular mechanisms of castration resistant prostate cancer. Oncol Lett 15: 6063-6076, 2018 .

5. Mottet N, Bellmunt J, Bolla M, Joniau S, Mason M, Matveev V, Schmid HP, Van der Kwast $T$, Wiegel $T$, Zattoni $F$ and Heidenreich A: EAU Guidelines on prostate cancer. Part II: Treatment of advanced, relapsing, and castration-resistant prostate cancer. Eur Urol 59: 572-583, 2011.

6. Summers N, Vanderpuye-Orgle J, Reinhart M, Gallagher M and Sartor O: Efficacy and safety of post-docetaxel therapies in metastatic castrate-resistant prostate cancer: A systematic review of the literature. Curr Med Res Opin 33: 1995-2008, 2017.

7. Seitz AK, Thoene S, Bietenbeck A, Nawroth $R$, Tauber $R$ Thalgott M, Schmid S, Secci R, Retz M, Gschwend JE, et al: AR-V7 in peripheral whole blood of patients with castration-resistant prostate cancer: Association with treatment-specific outcome under abiraterone and enzalutamide. Eur Urol 72: 828-834, 2017.

8. Berthold DR, Pond GR, Roessner M, de Wit R, Eisenberger M and Tannock AI; TAX-327 investigators: Treatment of hormone-refractory prostate cancer with docetaxel or mitoxantrone: Relationships between prostate-specific antigen, pain, and quality of life response and survival in the TAX-327 Study. Clin Cancer Res 14: 2763-2767, 2008.

9. Zhao Y, Huang H, Chen C, Liu H, Liu H, Su F, Bi J, Lam TB, Li J, Lin T and Huang J: Efficacy and safety of different interventions in castration resistant prostate cancer progressing after docetaxel-based chemotherapy: Bayesian network analysis of randomized controlled trials. J Cancer 9: 690-701, 2018.

10. Hongo H, Kosaka T and Oya M: Analysis of cabazitaxel-resistant mechanism in human castration-resistant prostate cancer. Cancer Sci 109: 2937-2945, 2018.

11. Yun EJ, Lo UG and Hsieh JT: The evolving landscape of prostate cancer stem cell: Therapeutic implications and future challenges. Asian J Urol 3: 203-210, 2016.

12. Faivre EJ, Wilcox D, Lin X, Hessler P, Torrent M, He W, Uziel T, Albert DH, McDaniel K, Kati W and Shen Y: Exploitation of castration-resistant prostate cancer transcription factor dependencies by the novel BET inhibitor ABBV-075. Mol Cancer Res 15: 35-44, 2017.

13. Zhu J, Sun C, Wang L, Xu M, Zang Y, Zhou Y, Liu X, Tao W, Xue B, Shan Y and Yang D: Targeting survivin using a combination of miR-494 and survivin shRNA has synergistic effects on the suppression of prostate cancer growth. Mol Med Rep 13. $1602-1610,2016$

14. Zhu J, Wang S, Zhang W, Qiu J, Shan Y, Yang D and Shen B: Screening key microRNAs for castration-resistant prostate cancer based on miRNA/mRNA functional synergistic network. Oncotarget 6: 43819-43830, 2015.
15. Yang DR, Ding XF, Luo J, Shan YX, Wang R, Lin SJ, Li G, Huang CK, Zhu J, Chen Y, et al: Increased chemosensitivity via targeting testicular nuclear receptor 4 (TR4)-Oct4-Interleukin 1 receptor antagonist (IL1Ra) axis in prostate cancer CD133+ Stem/progenitor cells to battle prostate cancer. J Biol Chem 288: 16476-16483, 2013.

16. Yu SJ, Yang L, Hong Q, Kuang XY, Di GH and Shao ZM: MicroRNA-200a confers chemoresistance by antagonizing TP53INP1 and YAP1 in human breast cancer. BMC Cancer 18: 74, 2018.

17. Yu G, Jia B, Cheng Y, Zhou L, Qian B, Liu Z and Wang Y: MicroRNA-429 sensitizes pancreatic cancer cells to gemcitabine through regulation of PDCD4. Am J Transl Res 9: 5048-5055, 2017.

18. Puhr M, Hoefer J, Schäfer G, Erb HH, Oh SJ, Klocker H, Heidegger I, Neuwirt H and Culig Z: Epithelial-to-mesenchymal transition leads to docetaxel resistance in prostate cancer and is mediated by reduced expression of miR-200c and miR-205. Am J Pathol 181: 2188-2201, 2012.

19. Zhang HL, Yang LF, Zhu Y, Yao XD, Zhang SL, Dai B, Zhu YP, Shen YJ, Shi GH and Ye DW: Serum miRNA-21: Elevated levels in patients with metastatic hormone-refractory prostate cancer and potential predictive factor for the efficacy of docetaxel-based chemotherapy. Prostate 71: 326-331, 2011.

20. Rokhlin OW, Scheinker VS, Taghiyev AF, Bumcrot D, Glover RA and Cohen MB: MicroRNA-34 mediates AR-dependent p53-induced apoptosis in prostate cancer. Cancer Biol Ther 7: 1288-1296, 2008.

21. Gov E and Arga KY: Interactive cooperation and hierarchical operation of microRNA and transcription factor crosstalk in human transcriptional regulatory network. IET Syst Biol 10: 219-228, 2016

22. Li H, Rokavec M, Jiang L, Horst D and Hermeking H: Antagonistic Effects of p53 and HIF1A on microRNA-34a regulation of PPP1R11 and STAT3 and Hypoxia-induced Epithelial to mesenchymal transition in colorectal cancer cells. Gastroenterology 153: 505-520, 2017.

23. Chang TH, Tsai MF, Gow CH, Wu SG, Liu YN, Chang YL, Yu SL, Tsai HC, Lin SW, Chen YW, et al: Upregulation of microRNA-137 expression by Slug promotes tumor invasion and metastasis of non-small cell lung cancer cells through suppression of TFAP2C. Cancer Lett 402: 190-202, 2017.

24. Mcmanus MT: MicroRNAs and cancer. Semin Cancer Biol 13: 253-258, 2003.

25. Gao L, Yang Y, Xu H, Liu R, Li D, Hong H, Qin M and Wang Y: MiR-335 functions as a tumor suppressor in pancreatic cancer by targeting OCT4. Tumour Biol 35: 8309-8318, 2014.

26. Livak KJ and Schmittgen TD: Analysis of relative gene expression data using real-time quantitative PCR and the 2(-Delta Delta C(T)) method. Methods 25: 402-408, 2001.

27. Kilkenny C, Browne WJ, Cuthill IC, Emerson $M$ and Altman DG: Improving bioscience research reporting: The ARRIVE guidelines for reporting animal research. PLoS Biol 8: e1000412, 2010.

28. Qiu X, Zhu J, Sun Y, Fan K, Yang DR, Li G, Yang G and Chang C: TR4 nuclear receptor increases prostate cancer invasion via decreasing the miR-373-3p expression to alter TGF $3 \mathrm{R} 2 / \mathrm{p}-\mathrm{Smad} 3$ signals. Oncotarget 6: 15397-15409, 2015.

29. Sachdeva M, Zhu S, Wu F, Wu H, Walia V, Kumar S, Elble R, Watabe K and Mo YY: p53 represses c-Myc through induction of the tumor suppressor miR-145. Proc Natl Acad Sci USA 106: 3207-3212, 2009.

30. Yin R, Zhang S, Wu Y, Fan X, Jiang F, Zhang Z, Feng D, Guo X and $\mathrm{Xu}$ L: microRNA-145 suppresses lung adenocarcinoma-initiating cell proliferation by targeting OCT4. Oncol Rep 25: 1747-1754, 2011

31. Ren D, Wang M, Guo W, Zhao X, Tu X, Huang S, Zou X and Peng X: Wild-type p53 suppresses the epithelial-mesenchymal transition and stemness in PC-3 prostate cancer cells by modulating miR-145. Int J Oncol 42: 1473-1481, 2013.

32. Cao L, Li C, Shen S, Yan Y, Ji W, Wang J, Qian H, Jiang X, Li Z, Wu M, et al: OCT4 increases BIRC5 and CCND1 expression and promotes cancer progression in hepatocellular carcinoma. BMC Cancer 13: 82, 2013

33. Zhang M, Latham DE, Delaney MA and Chakravarti A: Survivin mediates resistance to antiandrogen therapy in prostate cancer. Oncogene 24: 2474-2482, 2005.

34. Gao M, Guo L, Wang H, Huang J, Han F, Xiang S and Wang J: Orphan nuclear receptor RORgamma confers doxorubicin resistance in prostate cancer. Cell Biol Int 44: 2170-2176, 2020. 
35. Wang Z, Li Y, Wu D, Yu S, Wang Y and Chan FL: Correction: Nuclear receptor $\mathrm{HNF} 4 \alpha$ performs a tumor suppressor function in prostate cancer via its induction of p21-driven cellular senescence. Oncogene 39: 6263, 2020.

36. Shen J, Lin H, Li G, Jin RA, Shi L, Chen M, Chang C and Cai X: TR4 nuclear receptor enhances the cisplatin chemo-sensitivity via altering the ATF3 expression to better suppress HCC cell growth. Oncotarget 7: 32088-32099, 2016.

37. Shyr CR, Hu YC, Kim E and Chang C: Modulation of estrogen receptor-mediated transactivation by orphan receptor TR4 in MCF-7 cells. J Biol Chem 277: 14622-14628, 2002.

38. Zhang L, Zhang J, Ma Y, Chen J, Dong B, Zhao W, Wang X, Zheng Q, Fang F and Yang Y: Testicular orphan receptor 4 (TR4) is a marker for metastasis and poor prognosis in non-small cell lung cancer that drives the EMT phenotype. Lung Cancer 89: 320-328, 2015 .

39. Bai J, Yeh S, Qiu X, Hu L, Zeng J, Cai Y, Zuo L, Li G, Yang G and Chang C: TR4 nuclear receptor promotes clear cell renal cell carcinoma (ccRCC) vasculogenic mimicry (VM) formation and metastasis via altering the miR490-3p/vimentin signals. Oncogene 37: 5901-5912, 2018.

40. Wang M, Sun Y, Xu J, Lu J, Wang K, Yang DR, Yang G, Li G and Chang C: Preclinical studies using miR-32-5p to suppress clear cell renal cell carcinoma metastasis via altering the miR-32-5p/TR4/HGF/Met signaling. Int J Cancer 143: 100-112, 2018.

41. Lin SJ, Lee SO, Lee YF, Miyamoto H, Yang DR, Li G and Chang C: TR4 nuclear receptor functions as a tumor suppressor for prostate tumorigenesis via modulation of DNA damage/repair system. Carcinogenesis 35: 1399-1406, 2014

42. Ding X, Yang DR, Lee SO, Chen YL, Xia L, Lin SJ, Yu S, Niu YJ, Li G and Chang C: TR4 nuclear receptor promotes prostate cancer metastasis via upregulation of CCL2/CCR2 signaling. Int J Cancer 136: 955-964, 2015.

43. Yu S, Wang M, Ding X, Xia L, Chen B, Chen Y, Zhang Z, Niu Y, Li G and Chang C: Testicular orphan nuclear receptor 4 is associated with the radio-sensitivity of prostate cancer. Prostate 75: 1632-1642, 2015.

44. Chen B, Yu S, Ding X, Jing C, Xia L, Wang M, Matro E, Rehman F, Niu Y, Li G and Chang C: The role of testicular nuclear receptor 4 in chemo-resistance of docetaxel in castration-resistant prostate cancer. Cancer Gene Ther 21: 411-415, 2014.

45. Li S, Wu X, Xu Y, Wu S, Li Z, Chen R, Huang N, Zhu Z and $\mathrm{Xu} X$ : miR-145 suppresses colorectal cancer cell migration and invasion by targeting an ETS-related gene. Oncol Rep 36: 1917-1926, 2016.

46. Xu Q, Liu LZ, Qian X, Chen Q, Jiang Y, Li D, Lai L and Jiang BH: miR-145 directly targets p70S6K1 in cancer cells to inhibit tumor growth and angiogenesis. Nuclc Acids Res 40: 761-774, 2012
47. Cho WC, Chow AS and Au JS: miR-145 inhibits cell proliferation of human lung adenocarcinoma by targeting EGFR and NUDT1. RNA Biol 8: 125-131, 2011.

48. Chiyomaru T, Enokida H, Tatarano S, Kawahara K, Uchida Y, Nishiyama K, Fujimura L, Kikkawa N, Seki N and Nakagawa M: miR-145 and miR-133a function as tumour suppressors and directly regulate FSCN1 expression in bladder cancer. $\mathrm{Br}$ J Cancer 102: 883-891, 2010.

49. Fuse M, Nohata N, Kojima S, Sakamoto S, Chiyomaru T, Kawakami K, Enokida H, Nakagawa M, Naya Y, Ichikawa T and Seki N: Restoration of miR-145 expression suppresses cell proliferation, migration and invasion in prostate cancer by targeting FSCN1. Int J Oncol 38: 1093-1101, 2011.

50. Gong P, Zhang T, He D and Hsieh JT: MicroRNA-145 modulates tumor sensitivity to radiation in prostate cancer. Radiat Res 184: 630-638, 2015.

51. Griesing S, Kajino T, Tai MC, Liu Z, Nakatochi M, Shimada Y, Suzuki M and Takahashi T: Thyroid transcription factor-1-regulated microRNA-532-5p targets KRAS and MKL2 oncogenes and induces apoptosis in lung adenocarcinoma cells. Cancer Sci 108: 1394-1404, 2017.

52. Pan Q, Meng L, Ye J, Wei X, Shang Y, Tian Y, He Y, Peng Z, Chen L, Chen W, et al: Transcriptional repression of miR-200 family members by Nanog in colon cancer cells induces epithelial-mesenchymal transition (EMT). Cancer Lett 392: 26-38, 2017.

53. O'Geen H, Lin YH, Xu X, Echipare L, Komashko VM, He D, Frietze S, Tanabe O, Shi L, Sartor MA, et al: Genome-wide binding of the orphan nuclear receptor TR4 suggests its general role in fundamental biological processes. BMC Genomics 11: 689, 2010.

54. Larne O, Hagman Z, Lilja H, Bjartell A, Edsjö A and Ceder Y: miR-145 suppress the androgen receptor in prostate cancer cells and correlates to prostate cancer prognosis. Carcinogenesis 36: 858-866, 2015.

55. Li R, Azzollini D, Shen R, Thorup J, Clasen-Linde E, Cortes D and Hutson JM: Postnatal germ cell development during first 18 months of life in testes from boys with non-syndromic cryptorchidism and complete or partial androgen insensitivity syndrome. J Pediatr Surg 54: 1654-1659, 2019.

56. Wang J, Lu M, Qiu C and Cui Q: TransmiR: A transcription factor-microRNA regulation database. Nucleic Acids Res 38 (Database Issue): D119-D122, 2010.

This work is licensed under a Creative Commons Attribution-NonCommercial-NoDerivatives 4.0 International (CC BY-NC-ND 4.0) License. 